\title{
TU/e Emonowe

\section{Performance analysis of wireless scheduling with ARQ in fast fading channels}

\section{Citation for published version (APA):}

Tan, H. P. (2005). Performance analysis of wireless scheduling with ARQ in fast fading channels. (Report Eurandom; Vol. 2005047). Eurandom.

\section{Document status and date:}

Published: 01/01/2005

\section{Document Version:}

Publisher's PDF, also known as Version of Record (includes final page, issue and volume numbers)

\section{Please check the document version of this publication:}

- A submitted manuscript is the version of the article upon submission and before peer-review. There can be important differences between the submitted version and the official published version of record. People interested in the research are advised to contact the author for the final version of the publication, or visit the $\mathrm{DOI}$ to the publisher's website.

- The final author version and the galley proof are versions of the publication after peer review.

- The final published version features the final layout of the paper including the volume, issue and page numbers.

Link to publication

\section{General rights}

Copyright and moral rights for the publications made accessible in the public portal are retained by the authors and/or other copyright owners and it is a condition of accessing publications that users recognise and abide by the legal requirements associated with these rights.

- Users may download and print one copy of any publication from the public portal for the purpose of private study or research.

- You may not further distribute the material or use it for any profit-making activity or commercial gain

- You may freely distribute the URL identifying the publication in the public portal.

If the publication is distributed under the terms of Article 25fa of the Dutch Copyright Act, indicated by the "Taverne" license above, please follow below link for the End User Agreement:

www.tue.nl/taverne

Take down policy

If you believe that this document breaches copyright please contact us at:

openaccess@tue.nl

providing details and we will investigate your claim. 


\title{
Performance Analysis of Wireless Scheduling with ARQ in Fast Fading Channels
}

\author{
Hwee Pink Tan \\ EURANDOM, Eindhoven University of Technology, P.O. Box 513, 5600 MB, \\ Eindhoven, The Netherlands \\ tan@eurandom.tue.nl
}

\begin{abstract}
In this paper, we study the performance of opportunistic scheduling for downlink data transmissions with type-II packet-combining hybrid ARQ in a multirate cellular network for a fast fading environment. We develop a Markov model for a two-user scenario with two feasible transmission rates, and derive the throughput distribution. $\mathrm{Nu}$ merical results suggest that there exists an operating region within which opportunistic scheduling maintains its scheduling gain over round-robin scheduling; this region disappears when the gap between the transmission rates becomes very large. In ongoing work, we seek to extend the analysis of the model as well as generalize the model to a multi-user scenario.
\end{abstract}

\section{Introduction}

Emerging multirate cellular networks are envisaged to provide high-rate data services to mobile users, in addition to traditional voice and low-rate data services. Since the channel quality of wireless links varies across the user population due to differences in path-loss, as well as fading effects, a variety of channel-aware or opportunistic scheduling approaches (e.g., [1]) have been studied recently that exploit this information in order to maximize channel efficiency. To facilitate the seamless operation of higher layer protocols such as TCP over such a network, link-layer protocols such as ARQ (automatic repeat request) are often used to improve the transmission reliability through packet retransmissions. While pure or hybrid type-I ARQ maintains the same probability of packet success at every transmission attempt, hybrid type-II packet-combining ARQ (e.g., [2]) effectively combines the soft decision values of the previous noisy copies to accomplish better probability of packet success.

While most prior work studied opportunistic scheduling and ARQ separately, few attempts have been made $[3,4]$ to consider the problems collectively. In [3], the authors considered hybrid type-I ARQ, and developed an analytical framework to derive both short-term and long-term performance measures for both uncorrelated and correlated wireless channels. In this work, perfect channel state information is assumed at the base station, which is justifiable in a slow fading channel, since channel prediction will be sufficiently accurate. On the other hand, in [4], the authors considered packet-combining type-II hybrid ARQ, 
where the probability of decoding failure depends on the number of transmission attempts as well as the user's channel condition. However, the dependence on the latter factor is removed by considering a slow fading environment, where each user's channel is constant over the time-scale of interest. An optimal scheduler was proposed that minimizes the total average cost, expressed as an increasing function of the queue length.

\subsection{Contribution of This Paper}

In this paper, we analyze the performance of an opportunistic scheduling scheme with packet-combining type-II hybrid ARQ in a fast fading environment. In such an environment, the analysis becomes more complex since the probability of decoding failure becomes a bivariate function. In addition, the assumption of perfect channel knowledge at the base station can no longer be justified, since channel prediction can be highly inaccurate in such an environment. Based on our analytical model for the scheduling and ARQ mechanism, numerical results show that under certain conditions, opportunistic scheduling actually loses its scheduling gain over round robin scheduling, which is channel-unaware.

The paper is organized as follows: In Section 2, we define the system model and the assumptions that we make. The analysis of the model is described in Section 3. In Section 4, we compare the performance of various scheduling schemes for different hybrid ARQ mechanisms. Finally, some concluding remarks are given in Section 5 .

\section{System Model and Assumptions}

We consider the downlink slotted transmission from a single base station to $M$ mobile users, where each fixed-size time slot is allocated to one user and the data flow corresponding to each mobile user is continuously backlogged. We characterize the channel condition of user $j$ in terms of (a) its feasible transmission rate (bits/slot), $t_{j}^{f}$, where $t_{\min } \leq t_{j}^{f} \leq t_{\max }$, and (b) the resulting probability of decoding failure, $p_{j}^{e}$, if it transmits. We consider a fast fading channel, where $t_{j}^{f}$ is assumed to be uniformly distributed over $\left[t_{\min }, t_{\max }\right]$ and independent in each slot.

\subsection{Hybrid ARQ Mechanism}

To characterize the mechanism of type-II packet-combining hybrid ARQ, let $t_{r_{j}, j}^{a}$ denote the actual transmission rate of user $j$ at the $\left(r_{j}+1\right)^{t h}$ transmission attempt. We consider the following cases:

$\boldsymbol{r}_{j}=\mathbf{0}$ For the first attempt, user $j$ will transmit at $t_{0, j}^{a}$ corresponding to the predicted feasible rate. For a fast fading channel, $t_{0, j}^{a}$ and $t_{j}^{f}$ will be identically distributed. 
$\boldsymbol{r}_{\boldsymbol{j}}>\mathbf{0}$ If the first attempt fails, subsequent transmissions will occur at the same rate as the previous attempt, i.e., $t_{r_{j}, j}^{a}=t_{r_{j}-1, j}^{a}$, and we expect the probability of decoding failure to be reduced with $r_{j}$, i.e., if $p_{j}^{e}=\alpha$ for $r_{j}=a$, then:

$$
p_{j}^{e}<\alpha \quad \text { if } r_{j}>a
$$

In addition to Eq. (1), it is reasonable to assume that if $p_{j}^{e}=\beta$ for $t_{r_{j}, j}^{a}=$ $t_{j}^{f}$, then the following properties should hold:

$$
p_{j}^{e}\left\{\begin{array}{l}
>\beta, t_{r_{j}, j}^{a}>t_{j}^{f} \\
\leq \beta, t_{r_{j}, j}^{a}<t_{j}^{f}
\end{array}\right.
$$

Hence, we propose the following model for $p_{j}^{e}$ that satisfies both Eq. (1) and (2):

$$
p_{j}^{e}= \begin{cases}\left(1+\frac{t_{\max \left(r_{j}-1,0\right), j}^{a}-t_{j}^{f}}{t_{\max }-t_{\min }}\right) \delta \cdot 0.5^{r_{j}}, & r_{j}<r_{\max } \\ 0, & r_{j}=r_{\max } .\end{cases}
$$

where $r_{\max }$ is the retransmission threshold such that if $r=r_{\max }$, the transmission is always successful, and $\delta$ is a constant that indicates channel quality, $0 \leq \delta \leq$ $\frac{1}{2 \cdot 0.5^{r_{m a x}}}$, so that $0 \leq p_{j}^{e}<1$. We note that Eq. (3) is also applicable to Type-I hybrid ARQ.

\subsection{Scheduling Mechanisms}

We consider an opportunistic scheduling mechanism which selects the mobile user $m^{*}$ with the least probability of decoding failure for transmission so as to maximize the overall system throughput, i.e.,

$$
m^{*}=\arg \min _{1 \leq j \leq M} p_{j}^{e}
$$

As a benchmark for comparison, we also consider a round-robin scheduling mechanism.

\section{Model Analysis}

In this section, we outline our model analysis for opportunistic scheduling with type-II packet-combining hybrid ARQ for a simple two-user scenario, where $r_{\max }=1$ and $t_{j}^{f} \in\left\{t_{\min }, t_{\max }\right\}$.

According to Eq. (4), to determine $m^{*}$ in each slot $i$, it is necessary to compute $p_{j}^{e} \forall j$, which in turn depends on the values of $r_{j}$ and $t_{\max \left(r_{j}-1,0\right), j}^{a}$. If we define $x_{j}=\left(r_{j}, t_{\max \left(r_{j}-1,0\right), j}^{a}\right)$ for user $j$ in any slot, then the scheduling and ARQ mechanisms can be characterized by $x \equiv\left(x_{1}, x_{2}\right) \in X$, where $X=\{(0,0)$, $\left.\left(1, t_{\min }, 0\right),\left(1, t_{\max }, 0\right),\left(0,1, t_{\min }\right),\left(0,1, t_{\max }\right)\right\}$. By considering all possible transmission events in each slot, we obtain the state transition diagram and the state transition probability matrix as shown in Fig. 1. 


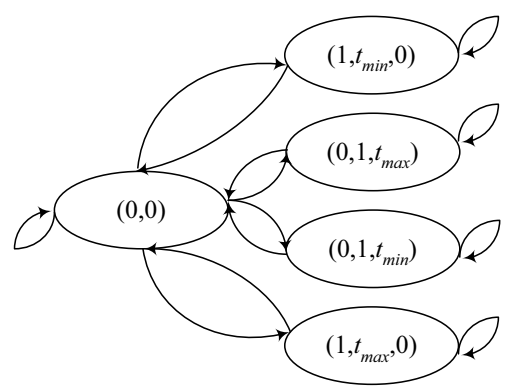

\begin{tabular}{|c|c|c|c|c|c|}
\hline & $(0,0)$ & $1, t_{m i n}$, & $\left(1, t_{\max }, 0\right.$ & $, 1, t_{m}$ & $0,1, t_{\max }$ \\
\hline$(0,0)$ & $1-5 \delta / 8$ & $\delta / 8$ & $3 \delta / 16$ & $\delta / 8$ & $3 \delta / 16$ \\
\hline$\left(1, t_{\text {min }}, 0\right)$ & $7 / 8$ & $1 / 8$ & 0 & 0 & 0 \\
\hline$\left(1, t_{\max }, 0\right)$ & $7 / 8$ & 0 & $1 / 8$ & 0 & 0 \\
\hline$\left(0,1, t_{\min }\right)$ & $7 / 8$ & 0 & 0 & $1 / 8$ & 0 \\
\hline$\left(0,1, t_{\max }\right)$ & $7 / 8$ & 0 & 0 & 0 & $1 / 8$ \\
\hline
\end{tabular}

Fig. 1. (Left) State-transition diagram and (right) state-transition probability matrix for two-user scenario with opportunistic scheduling.

We observe that given the value of $x$ in slot $i$-1, its pmf in slot $i$ can be obtained. Hence, we obtain a finite-state ergodic Markov chain with state variable, $x$, observed at each slot interval, with state space $X$ as defined above. From the state transition probability matrix in Fig. ??, the steady-state pmf of $x$ can be computed as follows:

$$
\operatorname{Prob}(x=X)=\left\{\begin{array}{l}
\frac{14}{14+10 \delta}, X=(0,0) \\
\frac{2 \delta}{14+10 \delta}, X=\left\{\left(1, t_{\text {min }}, 0\right),\left(0,1, t_{\text {min }}\right)\right\} \\
\frac{3 \delta}{14+10 \delta}, X=\left\{\left(1, t_{\text {max }}, 0\right),\left(0,1, t_{\text {max }}\right)\right\}
\end{array}\right.
$$

We can obtain expressions for the pmf of each user's throughput, conditioned on $x$. Then, using Eq. (5), we obtain the pmf of each user's throughput, $t$, as follows:

$$
\operatorname{Prob}(t=T)=\left\{\begin{array}{l}
\frac{77+10 \delta}{16(14+10 \delta)}, T=t_{\min } \\
\frac{35}{16(14+10 \delta)}, T=t_{\max } \\
\frac{56+75 \delta}{8(14+10 \delta)}, T=0
\end{array}\right.
$$

The analysis proceeds in a similar manner with round-robin scheduling, type-I hybrid ARQ and type-II hybrid ARQ with other values of $r_{\max }$.

\section{Performance Evaluation}

In this section, we evaluate the mean throughput $\left(\mu_{t}\right)$ as well as throughput fluctuation $\left(\sigma_{t}\right)$ of opportunistic scheduling $(\mathbf{O S})$ and round-robin scheduling (RR) with Type-I and Type-II packet-combining hybrid ARQ with $r_{\max }=2$ based on Section 3. The results are shown in Fig. 2 for $t_{\min }=100$ and $t_{\max }=2 t_{\min }$.

We note that for sufficiently large $\delta, \mu_{t}^{O S}>\mu_{t}^{R R}$, i.e., OS maintains its scheduling gain over $\mathbf{R R}$ as expected. However, as $\delta$ is reduced below some threshold $\delta^{\mu_{t}}$, this scheduling gain is lost. Similarly, there exists a corresponding threshold for throughput fluctuation (denoted by $\delta^{\sigma_{t}}$ ) such that when $\delta<\delta^{\sigma_{t}}$, 
the throughput of $\mathbf{O S}$ is less jittery than that of $\mathbf{R R}$ and vice versa. Since $\delta^{\mu_{t}}<\delta^{\sigma_{t}}$, we can define the region $\delta^{\mu_{t}} \leq \delta \leq \delta^{\sigma_{t}}$ where OS is the preferred scheduling scheme with higher and less jittery throughput; beyond this region, there is a trade-off between $\mathbf{O S}$ and $\mathbf{R R}$ in terms of $\mu_{t}$ and $\sigma_{t}$.

The corresponding results for the asymptotic case where $t_{\max }>t_{\min }$ are shown in Fig. 3. As the gap between the feasible rates, $t_{\text {max }}-t_{\text {min }}$, widens, both $\delta^{\mu_{t}}$ and $\delta^{\sigma_{t}}$ increase such that for the asymptotic case, $\delta^{\mu_{t}}=\delta^{\sigma_{t}}=\delta_{\max }$. In this case, RR achieves higher but more jittery throughput than OS.
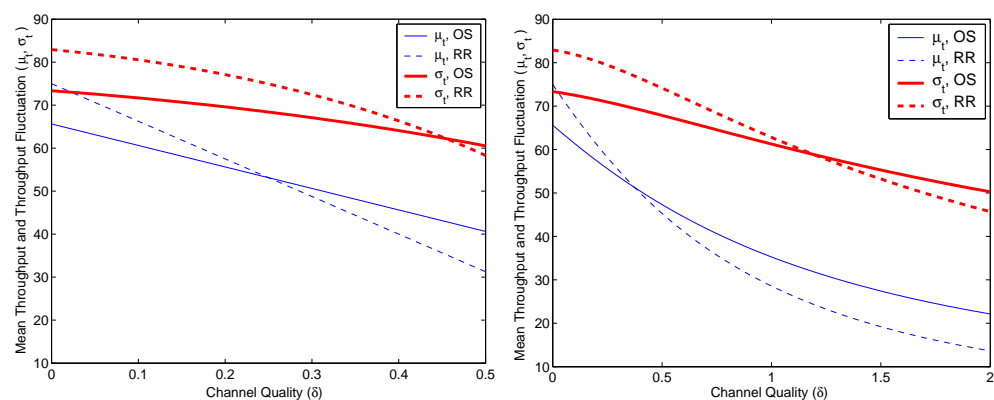

Fig. 2. Performance comparison of Opportunistic and Round-Robin Scheduling for two-user scenario in a fast fading channel with $t_{\min }=100$ and $t_{\max }=2 t_{\min }$ for various $\delta$ with hybrid (left) Type-I and (right) Type-II $\left(r_{\max }=2\right)$ hybrid ARQ.
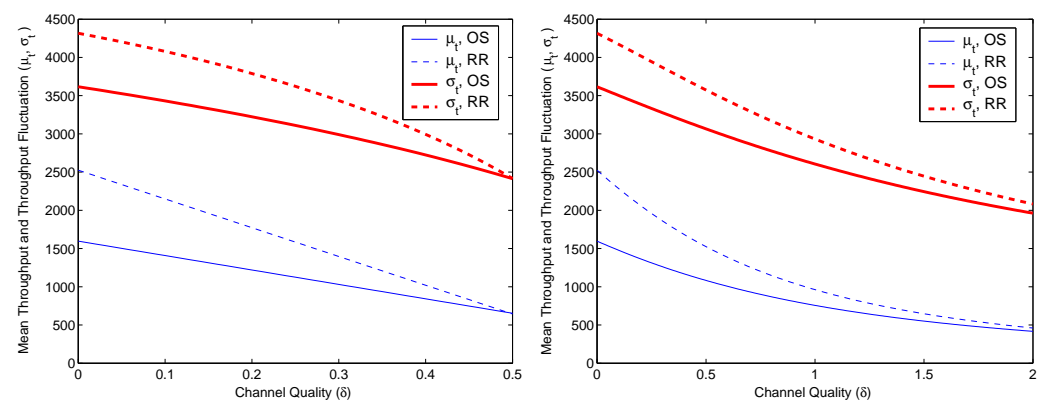

Fig. 3. Performance comparison of Opportunistic and Round-Robin Scheduling for two-user scenario in a fast fading channel with $t_{\max }>t_{\min }$ for various $\delta$ with hybrid (left) Type-I and (right) Type-II $\left(r_{\max }=2\right)$ hybrid ARQ. 


\section{Conclusions}

In this paper, we analyze the performance of opportunistic scheduling with typeII packet-combining hybrid ARQ for downlink data transmissions in a multirate cellular network for a fast fading environment. By defining the number of transmission attempts as well as the transmission rate of the previous attempt as state variables, we model the scheduling and ARQ mechanism as a Markov chain. By analyzing the Markov model for a two-user scenario with two feasible transmission rates, we derive the throughput distribution for each user.

Numerical results suggest that there exists an operating region within which opportunistic scheduling maintains its scheduling gain over round-robin scheduling, which is channel-unaware. In the asymptotic case where the gap between the transmission rates becomes very large, this region disappears and round-robin scheduling achieves a higher throughput at the expense of higher throughput fluctuation. In ongoing work, we seek to expand the current analysis and investigate if the existing observations can be generalized to a more general scenario.

\section{References}

1. Liu, X., Chan, E.K.P., Shroff, N.B.: Opportunistic Transmission Scheduling with Resource-Sharing Constraints in Wireless Networks. IEEE Journal of Selected Areas in Communications, 19(29):2053-2064, October 2001.

2. Harvey, B.A., Wicker, S.B.: Packet Combining Systems Based on the Viterbi Decoder. IEEE Transactions on Communications, 42(4):1544-1557, April 1994.

3. Issariyakul, T., Hossain, E.: Channel-Quality-Based Opportunistic Scheduling with ARQ in Multi-Rate Wireless Networks: Modeling and Analysis, Submitted to IEEE Transactions on Wireless Communications, 2004.

4. Huang, J., Berry, R., Honig, M.L.: Wireless Scheduling with Hybrid ARQ, $38^{\text {th }}$ Conference on Information Sciences and Systems, March 2004. 